\title{
Curvularia ahvazensis and $C$. rouhanii spp. nov. from Iran
}

\author{
Mehrabi-Koushki $\mathbf{M}^{1,2 *}$, Pooladi $P^{1,3}$, Eisvand $P^{1,3}$ and Babaahmadi $G^{1}$ \\ ${ }^{1}$ Plant Protection Department, Agriculture Faculty, Shahid Chamran University of Ahvaz, Ahvaz, Iran \\ ${ }^{2}$ Biotechnology and Bioscience Research Center, Shahid Chamran University of Ahvaz, Ahvaz, Iran \\ ${ }^{3}$ The second and third authors contributed equally to the study.
}

Mehrabi-Koushki M, Pooladi P, Eisvand P, Babaahmadi G 2018 - Curvularia ahvazensis and $C$. rouhanii spp. nov. from Iran. Mycosphere 9(6), 1173-1186, Doi 10.5943/mycosphere/9/6/7

\begin{abstract}
Three isolates of Curvularia were obtained from diseased Eucalyptus sp., Syngonium vellozianum and Zinnia elegans. Phylogenetic trees inferred from combined dataset of the internal transcribed spacer ribosomal DNA (ITS), partial glyceraldehyde-3-phosphate dehydrogenase $(\mathrm{GPDH})$, and partial translation elongation factor 1- $\alpha$ (EF1 $\alpha)$ along with morphological characterization, showed they are two novel species. Curvularia ahvazensis sp. nov. and $C$. rouhanii sp. nov. are described and illustrated and shown to be morphologically distinct from previously known species of Curvularia.
\end{abstract}

Key words - Two new species - Common zinnia - Eucalyptus - syngonium - multi-locus phylogenetic analysis

\section{Introduction}

Helminthosporium-like fungi associated with Cochliobolus, Helminthosporium, Bipolaris and Curvularia, contains some of the most highly damaging plant pathogens (Webster \& Webber 2007). These genera produce dark coloured multi-septate conidia and belong to the family Pleosporaceae, class Dothideomycetes (Hibbett 2007, Wijayawardene et al. 2017, 2018). Curvularia sensu lato (Boedijn 1933) is generally characterized by curved and euseptate phragmoconidia with an unevenly swollen central cell, usually less strongly pigmented end cells, and a protuberant hilum in some species; with blastic-sympodial and tretic conidiogenesis. Recently, Curvularia species have been subjected to multigene phylogenetic analyses in combination with morphology (Manamgoda et al. 2012a, b, 2014, 2015, Tan et al. 2014, 2018, Tomaso-Peterson et al. 2016, Marin-Felix et al. 2017a, b, Heidari et al. 2018, Liang et al. 2018). As a result of this, several Bipolaris species are now accepted in the genus Curvularia (Manamgoda et al. 2012a, b, 2014, 2015, Tan et al. 2014, 2018, Tomaso-Peterson et al. 2016). The partial regions of ITS (internal nuclear ribosomal transcribed spacer region), GPDH (glyceraldehyde-3-phosphate dehydrogenase) and EF1 $\alpha$ (translation elongation factor 1-a) were used exclusively or in combination for the species delimitation of Curvularia (Manamgoda et al. 2012a, b, 2014, 2015, Tan et al. 2014, 2018, Tomaso-Peterson et al. 2016, Marin-Felix et al. 2017a, b, Heidari et al. 2018, Liang et al. 2018). Including the most recent new species descriptions (Marin-Felix et al. 2017a, b, Heidari et al. 2018, Tan et al. 2018, Liang et al. 2018), Curvularia sensu stricto includes 92 validated species with very wide morphological variation (http://www.mycobank.org). 
In the present study, two new species of Curvularia isolated in Iran are described and illustrated through a polyphasic approach. A multi-locus phylogeny (ITS, GPDH and EF1 $\alpha$ ), combined with morphological analysis, was used to delimit them from previously described species.

\section{Material and Methods}

\section{Isolates and purification}

During 2015, symptomatic plants of Eucalyptus sp. (with stem canker and die-back), Zinnia elegans (with wilting and root rot) and Syngonium vellozianum (with leaf blight) were sampled from Ahvaz and Behbahan in the southwest of Iran. Small tissue pieces $(0.3-1 \mathrm{~cm})$ were cut from the interface between healthy and diseased tissue and surface-disinfected for 2-4 min in a solution containing $2 \%(\mathrm{v} / \mathrm{v})$ sodium hypochlorite. They were then rinsed three times in sterile distilled water ( $2 \mathrm{~min}$ ) and air-dried in a microbiological hood (Alexopoulos \& Beneke 1962). Plant pieces were then plated on potato dextrose agar (PDA) supplemented with streptomycin $(30 \mathrm{mg} / \mathrm{L})$ and incubated at $28{ }^{\circ} \mathrm{C}$ for 5-10 days. Following incubation, three isolates having distoseptate phragmoconidia were obtained and shown to be two new species of Curvularia. The type specimens (dried cultures) of the new species are deposited in Herbarium Ministerii Iranici Agriculturae, Iranian Research Institute of Plant Protection, Tehran, Iran. Ex-type living cultures are deposited in IRAN (Iranian Fungal Culture Collection, Iranian Research Institute of Plant Protection, Iran), CBS (Centraalbureau voor Schimmelcultures, Utrecht, The Netherlands) and SCUA (Collection of Fungal Cultures, Department of Plant Protection, Shahid Chamran University of Ahvaz, Iran).

\section{Growth characterization and morphological observations}

Growth rates and colony characteristics were determined on PDA (Merck) and corn meal agar (CMA, Sigma Aldrich) at three different temperatures $\left(28,35\right.$ and $\left.40{ }^{\circ} \mathrm{C}\right)$ in a 12 -h fluorescent light-dark regime. The colony diameter was measured daily for at least eight days. Sporulation structures (including conidia, conidiophores and conidiogenous cells) were examined and measured using 40x and 100x objective lens of a Leitz Wetzlar (SM-LUX) Basic Biological light microscope. Slide cultures were prepared according to the protocol described by Beneke \& Rogers (1996). Measurements are reported as a maximum and minimum range in association with $95 \%$ confidence limits and standard deviation. Images were prepared and recorded with an OLYMPUS BX51 microscope fitted with an OLYMPUS DP12 digital camera.

\section{Biomass preparation, DNA extraction and amplification}

Mycelial biomass was recovered from cultures grown in Erlenmeyer flasks containing potatodextrose-broth medium (PDB) and passed through filter papers. The biomass was freeze-dried (Freeze-Dryer, Alpha 1-2LD Plus, Christ) and powdered in a mortar containing liquid nitrogen. Genomic DNA was extracted from approximately $100 \mathrm{mg}$ of freeze-dried mycelium using an organic method described by Raeder \& Broda (1985), with some modification (Ahmadpour et al. 2017). A part of nuclear DNA, containing the ITS $1 \& 2,5.8 \mathrm{~S}$ and LSU-D1/D2 regions, was amplified by PCR using the primer combinations ITS1 and NL4 (White et al. 1990, O'Donnell 1993). A fragment of the GPDH gene was amplified using the primer pair gpd1 and gpd2 (Berbee et al. 1999). A partial region of EF1 $\alpha$ was amplified using the primer pair EF1-983 and EF1-2218R (Schoch et al. 2009). Each polymerase chain reaction (PCR) was performed in a $50 \mu 1$ reaction volume which contained $5 \mu \mathrm{l} 10 \times$ PCR buffer, $1.6 \mu \mathrm{l}$ of each primer $(10 \mu \mathrm{M}), 6 \mu \mathrm{MgCl}_{2}(25 \mathrm{mM})$, $1 \mu \mathrm{l}$ template DNA, and $0.25 \mu \mathrm{l}$ Taq DNA polymerase (5 units/ $\mu 1$, GenetBio, South Korea). Amplicons were produced in an automated thermal cycler (MJ Mini ${ }^{\mathrm{TM}}$ Gradient Thermal Cycler) with an initial melting step at $95^{\circ} \mathrm{C}$ for $3 \mathrm{~min}$, followed by 35 cycles of $30 \mathrm{~s}$ denaturation at $94{ }^{\circ} \mathrm{C}$, 40 s primers annealing at $54{ }^{\circ} \mathrm{C}(\mathrm{ITS}), 56{ }^{\circ} \mathrm{C}(\mathrm{GPDH})$ or $62{ }^{\circ} \mathrm{C}(\mathrm{EF} 1 \alpha), 1$ min extension at $72{ }^{\circ} \mathrm{C}$, 
and a 10 min extension at $72{ }^{\circ} \mathrm{C}$. PCR products of the expected size were purified by GF-1 AmbiClean Kit (Vivantis, Malaysia) according to the manufacturer's instructions.

\section{Sequencing, DNA analysis and phylogeny}

Amplicons were sequenced in both directions using the original PCR primers by Macrogen Company (Humanizing Genomics, Macrogen, South Korea). The obtained sequences were edited and assembled using BioEdit Sequence Alignment Editor Version 7.0.9.0 (Hall 1999) and DNA Baser Sequence Assembler v4 programs (2013, Heracle BioSoft, www.DnaBaser.com), respectively. The generated sequences were deposited in GenBank (Table 1). To study the phylogeny of Curvularia including the isolates under study and many of the known species, two combined matrix, containing ITS and GPDH (two-locus) and ITS, GPDH and EF1 $\alpha$ (three-locus) sequences, were constructed. Bipolaris drechsleri was selected as outgroup taxon. Sequences were aligned with Clustal W in MEGA version 6 (Tamura et al. 2013) and manually refined in BioEdit 7.0.9.0 (Hall 1999). Maximum likelihood (ML) analyses of the combined datasets was performed with MEGA version 6, using best-fitting nucleotide substitution model, as suggested by ML model test, subtree-pruning-regrafting (SPR) algorithm and following options: gaps (insertion/deletions) were included in analysis, bootstrap (BP) analyses were done with 1000 replicates, initial trees for ML were made by NJ/BioNJ algorithm and branch swap filter was set very strong. Statistical support of nodes of the phylogenetic trees is indicated as ML bootstrap values (BS) $=50-100 \%$. Two combined matrix used for phylogenetic analysis were deposited in the Treebase (http://purl.org/phylo/treebase/phylows/study/TB2: S22741).

\section{Results}

\section{Sequences and phylogenetic analysis}

The final Curvularia alignment to construct phylogenetic trees included 121 strains (Table 1), representing many of the known Curvularia species, two new species described in the present study and the outgroup taxon. The combined matrix consisted of 1652 characters including alignment gaps (ITS: 468, GPDH: 501, and EF1 $\alpha$ : 683). The number of conserved sites was 1172 (ITS: 327 , GPDH: 294, and EF1 $\alpha$ : 551). The number of variable and parsimony uninformative sites was 74 (ITS: 26, GPDH: 20, and EF1 $\alpha$ : 28) and 386 sites were variable and parsimony informative (ITS: 101, GPDH: 181, and EF1 $\alpha$ : 104). The best models of evolution calculated with MEGA 6 were the TN93 (Tamura-Nei) and GTR (General Time Reversible) with Invariant Site and Gamma Distribution $(\mathrm{G}+\mathrm{I})$ used for ML analysis of two- and three- locus datasets, respectively. In both two- and three- locus phylogenetic trees inferred from the combined dataset of ITS-GPDH and ITSGPDH-EF1 $\alpha$ (Fig. 1), both strains (CBS 144674 and CBS 144675) of C. rouhanii cluster together with BS $73 \%$ and $93 \%$ support, respectively. This species also associated with two other taxa, $C$. buchloes and C. spicifera, in a larger clade with BS 94\% support (Fig. 1). In three-locus phylogenetic tree, C. ahvazensis clustered with $C$. hawaiiensis into a distinct clade with BS $92 \%$ support. Two species, $C$. ahvazensis and $C$. hawaiiensis, could also be clearly distinguished based on morphology. The phylogenetic tree based on ITS-GPDH dataset (not shown) was constructed to show delimitation of some Curvullaria species for which their EF1 $\alpha$ sequences were not available.

\section{Taxonomy}

Curvularia ahvazensis M. Mehrabi-Koushki \& G. Babaahmadi, sp. nov.

Fig. 2 MycoBank: MB823459

Typification - IRAN, KHUZESTAN PROVINCE: Ahvaz. On rotten roots of common zinnia (Zinnia elegans), December 2015, G. Babaahmadi and M. Mehrabi-Koushki (holotype: IRAN 16942F, ex-type cultures: CBS $144673=$ IRAN 3136C = SCUA-1bi).

Etymology - Name refers to the city Ahvaz, where the fungus was collected. 
Morphology on PDA - Hyphae hyaline to pale brown, branched, septate, 2.5-5 $\mu \mathrm{m}$ diam. Conidiophores arising singly, septate, generally equal width in basal and median parts, upper part wider, straight or flexuous, sometimes geniculate at apex, mostly un-branched, sometimes bifurcated, cell walls thicker than those of vegetative hyphae, brown, darker towards apex, (7.5) $12-137.5(-187) \times 2-4 \mu \mathrm{m}, 95 \%$ confidence limits $=37.3-53.8 \times 2.7-3.1 \mu \mathrm{m},(\overline{\mathrm{x}} \pm \mathrm{SD}=45.5 \pm$ $36.9 \times 2.9 \pm 0.7 \mu \mathrm{m}, \mathrm{n}=45)$. Conidiogenous cells integrated or discrete, smooth or with verruculose nodes, terminal or intercalary, proliferating sympodially, with circular and slightly thickened scars, pale brown to brown, subcylindrical to slightly swollen. Conidia smooth-walled, straight or very-slightly curved, ellipsoid, oblong or cylindrical, tapering towards rounded ends, pale brown to brown, 2-6-distoseptate, end cells not paler, $(10-) 13.5-27(-35) \times(2.5-) 5-7.8 \mu \mathrm{m}$, $95 \%$ confidence limits $=20.6-22.1 \times 6.4-6.7 \mu \mathrm{m},(\overline{\mathrm{x}} \pm \mathrm{SD}=21.4 \pm 3.7 \times 6.5 \pm 0.7 \mu \mathrm{m}, \mathrm{n}=50)$. Hila slightly protruding, darkened, thickened, 1.5-3 $\mu \mathrm{m}$ diam. Chlamydospore and sexual morph not observed.

Table 1 Strains used in this study and their GenBank accession numbers. The new species are indicated in bold.

\begin{tabular}{|c|c|c|c|c|c|c|}
\hline \multirow{2}{*}{ Species } & \multirow{2}{*}{ Strain no. ${ }^{*}$} & \multirow{2}{*}{ Source } & \multirow{2}{*}{ Origin } & \multicolumn{3}{|c|}{ GenBank Accession numbers } \\
\hline & & & & ITS & GPDH & EF1a \\
\hline B. drechsleri & MUS0028 & $\begin{array}{l}\text { Microstegium } \\
\text { vimineum }\end{array}$ & USA & KF500532 & KF500535 & KM093761 \\
\hline C. aeria & $\begin{array}{l}\text { BRIP } \\
61232 b\end{array}$ & Oryza sativa & Australia & KU552200 & KU552162 & KU552155 \\
\hline C. affinis & CBS 154.34 & Unknown & Indonesia & KJ909780 & KM230401 & KM196566 \\
\hline C.ahvazensis & $\begin{array}{l}\text { CBS } \\
\text { 144673; } \\
\text { IRAN } \\
\text { 3136C; } \\
\text { SCUA-1bi }\end{array}$ & Zinnia elegans & Iran & KX139029 & MG428693 & MG428686 \\
\hline C. akaii & CBS 317.86 & $\begin{array}{l}\text { Themada } \\
\text { triandra }\end{array}$ & Japan & KJ909782 & KM230402 & KM196569 \\
\hline C. akaiiensis & BRIP 16080 & - & - & KJ415539 & KJ415407 & KJ415453 \\
\hline C. alcornii & $\begin{array}{l}\text { MFLUCC } \\
100703\end{array}$ & Zea & Thailand & JX256420 & JX276433 & JX266589 \\
\hline C. americana & $\begin{array}{l}\text { UTHSC 08- } \\
3414\end{array}$ & Homo sapiens & USA & HE861833 & HF565488 & - \\
\hline C. asianensis & $\begin{array}{l}\text { MFLUCC } \\
100711\end{array}$ & Panicum sp. & Thailand & JX256424 & JX276436 & JX266593 \\
\hline C. australiensis & BRIP 12044 & Oryza sativa & - & KJ415540 & KJ415406 & KJ415452 \\
\hline C. australiensis & CBS 172.57 & Oryza sativa & Vietnam & JN601026 & JN601036 & JN601003 \\
\hline C. australis & $\begin{array}{l}\text { BRIP } \\
12247 \mathrm{a}\end{array}$ & $\begin{array}{l}\text { Eragrostis } \\
\text { cilianensis }\end{array}$ & Australia & KC424609 & KC747759 & KC503954 \\
\hline C. australis & BRIP 12521 & $\begin{array}{l}\text { Sporobolus } \\
\text { carolii }\end{array}$ & - & KJ415541 & KJ415405 & KJ415451 \\
\hline C. bannonii & BRIP 16732 & $\begin{array}{l}\text { Jacquemontia } \\
\text { tamnifolia }\end{array}$ & USA & KJ415542 & KJ415404 & KJ415450 \\
\hline C. beasleyi & BRIP 10972 & Chloris gayana & Australia & MH414892 & MH433638 & MH433654 \\
\hline C. beasleyi & BRIP 16732 & $\begin{array}{l}\text { Leersia } \\
\text { hexandra }\end{array}$ & Australia & MH414893 & MH433639 & MH433655 \\
\hline $\begin{array}{l}\text { C. } \\
\text { beerburrumensis }\end{array}$ & BRIP 12942 & $\begin{array}{l}\text { Eragrostis } \\
\text { bahiensis }\end{array}$ & Australia & MH414894 & MH433634 & MH433657 \\
\hline $\begin{array}{l}\text { C. } \\
\text { beerburrumensis }\end{array}$ & BRIP 12555 & $\begin{array}{l}\text { Eragrostis } \\
\text { sororia }\end{array}$ & Australia & MH414895 & MH433640 & MH433656 \\
\hline C. boeremae & IMI 164633 & $\begin{array}{l}\text { Portulaca } \\
\text { oleracea }\end{array}$ & India & MH414911 & MH433641 & - \\
\hline C. borreriae & AR5176r & $\begin{array}{l}\text { Sorghum } \\
\text { bicolor }\end{array}$ & $\begin{array}{l}\text { South } \\
\text { Africa }\end{array}$ & KP400637 & KP419986 & KP735690 \\
\hline
\end{tabular}


Table 1 Continued.

\begin{tabular}{|c|c|c|c|c|c|c|}
\hline \multirow[t]{2}{*}{ Species } & \multirow[t]{2}{*}{ Strain no." } & \multirow[t]{2}{*}{ Source } & \multirow[t]{2}{*}{ Origin } & \multicolumn{3}{|c|}{ GenBank Accession numbers } \\
\hline & & & & ITS & GPDH & EF1a \\
\hline C. borreriae & $\begin{array}{l}\text { MFLUCC } \\
11-0422\end{array}$ & Unknown grass & Thailand & KP400638 & KP419987 & KM196571 \\
\hline C. bothriochloae & BRIP 12522 & Bothriochloa & Australia & KJ415543 & KJ415403 & KJ415449 \\
\hline C. brachyspora & CBS 186.50 & Soil & India & KJ922372 & KM061784 & KM230405 \\
\hline C. buchloes & CBS 246.49 & $\begin{array}{l}\text { Buchloe } \\
\text { dactyloides }\end{array}$ & USA & KJ909765 & KM061789 & KM196588 \\
\hline C. caricapapayae & CBS 135941 & Carica papaya & India & HG778984 & HG779146 & - \\
\hline C. chlamydospora & $\begin{array}{l}\text { UTHSC } \\
072764\end{array}$ & Toe nail & USA & HG779021 & HG779151 & - \\
\hline C. clavata & BRIP:61680 & Oryza sp. & Australia & KU552205 & KU552167 & KU552159 \\
\hline C. coatesiae & BRIP 24261 & Litchi chinensis & Australia & MH414897 & MH433636 & MH433659 \\
\hline C. coatesiae & BRIP 24170 & Air & Australia & MH414896 & MH433635 & MH433658 \\
\hline C. coicis & CBS 192.29 & Coix lacryma & Japan & JN192373 & JN600962 & JN601006 \\
\hline C. colbranii & BRIP 13066 & $\begin{array}{l}\text { Crinum } \\
\text { zeylanicum }\end{array}$ & Australia & MH414898 & MH433642 & MH433660 \\
\hline C. crustacea & BRIP 13524 & Sporobolus sp. & Indonesia & KJ415544 & KJ415402 & KJ415448 \\
\hline C. dactyloctenicola & CPC 28810 & $\begin{array}{l}\text { Dactyloctenium } \\
\text { aegyptium }\end{array}$ & Thailand & MF490815 & MF490837 & MF490858 \\
\hline C. dactyloctenii & BRIP 12846 & $\begin{array}{l}\text { Dactyloctenium } \\
\text { radulans }\end{array}$ & Australia & KJ415545 & KJ415401 & KJ415447 \\
\hline C. ellisii & CBS 193.62 & Air & Pakistan & JN192375 & JN600963 & JN601007 \\
\hline C. eragrosticola & BRIP 12538 & $\begin{array}{l}\text { Eragrostis } \\
\text { pilosa }\end{array}$ & Australia & MH414899 & MH433643 & MH433661 \\
\hline C. eragrostidis & CBS 189.48 & - & - & HG778986 & HG779154 & - \\
\hline C. geniculata & CBS 187.50 & Unknown seed & Indonesia & KJ909781 & KM083609 & KM230410 \\
\hline C. gladioli & ICMP 6160 & Gladiolus sp. & $\begin{array}{l}\text { New } \\
\text { Zealand }\end{array}$ & JX256426 & JX276438 & JX266595 \\
\hline C. graminicola & $\begin{array}{l}\text { BRIP } \\
23186 a\end{array}$ & - & Australia & JN192376 & JN600964 & JN601008 \\
\hline C. harveyi & BRIP 57412 & $\begin{array}{l}\text { Triticum } \\
\text { aestivum }\end{array}$ & Australia & KJ415546 & KJ415400 & KJ415446 \\
\hline C. hawaiiensis & BRIP 11987 & Oryza sativa & USA & KJ415547 & KJ415399 & KJ415445 \\
\hline $\begin{array}{l}\text { C. } \\
\text { heteropogonicola }\end{array}$ & BRIP 14579 & $\begin{array}{l}\text { Heteropogon } \\
\text { contortus }\end{array}$ & India & KJ415548 & KJ415398 & KJ415444 \\
\hline C. heteropogonis & CBS 284.91 & $\begin{array}{l}\text { Heteropogon } \\
\text { contortus }\end{array}$ & Australia & JN192379 & JN600969 & JN601013 \\
\hline C. hominis & AR 5118 & Lolium perene & USA & KP400639 & KP419988 & KM196580 \\
\hline C. hominis & $\begin{array}{l}\text { MFLUCC } \\
120191\end{array}$ & Unknown grass & Thailand & KP400640 & KP419989 & KM196581 \\
\hline C. homomorpha & CBS 156.60 & - & - & JN192380 & JN600970 & JN601014 \\
\hline C. inaequalis & CBS 102.42 & Sand dune soil & France & KJ922375 & KM061787 & KM196574 \\
\hline C. inaequalis & $\begin{array}{l}\text { DAOM } \\
20022\end{array}$ & Pisum sativum & Canada & KJ922374 & KM061786 & KM196575 \\
\hline C. intermedia & B19 & $\begin{array}{l}\text { Polygonatum } \\
\text { sibiricum }\end{array}$ & China & MF370184 & MF370185 & MF370186 \\
\hline C. ischaemi & CBS 630.82 & $\begin{array}{l}\text { Ischaemum } \\
\text { indicum }\end{array}$ & Fiji & JX256428 & JX276440 & - \\
\hline C. kenpeggii & BRIP 14530 & $\begin{array}{l}\text { Triticum } \\
\text { aestivum }\end{array}$ & Australia & MH414900 & MH433644 & MH433662 \\
\hline C. lamingtonensis & BRIP 12259 & $\begin{array}{l}\text { Microlaena } \\
\text { stipoides }\end{array}$ & Australia & MH414901 & MH433645 & MH433663 \\
\hline C. lunata & CBS 730.96 & lung biopsy & USA & JX256429 & JX276441 & JX266596 \\
\hline C. malina & CBS 131274 & Zoysia grass & USA & JF812154 & KP153179 & KR493095 \\
\hline C. malina & FLS-119 & Bermuda grass & USA & KR493070 & KR493083 & KR493093 \\
\hline
\end{tabular}


Table 1 Continued.

\begin{tabular}{|c|c|c|c|c|c|c|}
\hline \multirow[t]{2}{*}{ Species } & \multirow[t]{2}{*}{ Strain no." } & \multirow[t]{2}{*}{ Source } & \multirow[t]{2}{*}{ Origin } & \multicolumn{3}{|c|}{ GenBank Accession numbers } \\
\hline & & & & ITS & GPDH & EF1a \\
\hline C. mebaldsii & BRIP 12900 & $\begin{array}{l}\text { Cynodon } \\
\text { tranvaalensis }\end{array}$ & Australia & MH414902 & MH433647 & MH433664 \\
\hline C. mebaldsii & BRIP 13983 & $\begin{array}{l}\text { Cynodon } \\
\text { dactylon }\end{array}$ & Australia & MH414903 & MH433646 & MH433665 \\
\hline C. microspora & GUCC 6272 & $\begin{array}{l}\text { Hippeastrum } \\
\text { striatum }\end{array}$ & China & MF139088 & MF139097 & MF139115 \\
\hline C. microspora & GUCC 6273 & $\begin{array}{l}\text { Hippeastrum } \\
\text { striatum }\end{array}$ & China & MF139089 & MF139098 & MF139116 \\
\hline C. miyakei & CBS197.29 & $\begin{array}{l}\text { Eragrostis } \\
\text { pilosa }\end{array}$ & Japan & KJ909770 & KM083611 & KM196568 \\
\hline C. mosaddeghii & $\begin{array}{l}\text { IRAN } \\
3131 \mathrm{C}\end{array}$ & $\begin{array}{l}\text { Syzygium } \\
\text { cumini }\end{array}$ & Iran & MG846737 & MH392155 & MH392152 \\
\hline C. mosaddeghii & $\begin{array}{l}\text { IRAN } \\
3123 \mathrm{C}\end{array}$ & $\begin{array}{l}\text { Vigna } \\
\text { unguiculata }\end{array}$ & Iran & MG971270 & MG975597 & MH392151 \\
\hline C. mиehlenbeckiae & MUS 0031 & Sorghum sp. & USA & KP400647 & KP419996 & KM196578 \\
\hline C. muehlenbeckiae & AR 5180 & Sorghum sp. & USA & KP400649 & KP419998 & - \\
\hline C. neergaardii & BRIP 12919 & Oryza sativa & Ghana & KJ415550 & KJ415397 & KJ415443 \\
\hline C. neergaardii & $\begin{array}{l}\text { DAOM } \\
228085\end{array}$ & Desert soil & Chile & KJ909784 & KM083615 & KM196593 \\
\hline C. neoindica & IMI 129790 & Brassica nigra & India & MH414910 & MH433649 & MH433667 \\
\hline C. nicotiae & BRIP 11983 & - & - & KJ415551 & KJ415396 & KJ415442 \\
\hline C. nodosa & СРC 28801 & $\begin{array}{l}\text { Urochloa } \\
\text { reptans }\end{array}$ & Thailand & MF490817 & MF490839 & MF490860 \\
\hline C. nodosa & CPC 28812 & $\begin{array}{l}\text { Chloris } \\
\text { barbata }\end{array}$ & Thailand & MF490818 & MF490840 & MF490861 \\
\hline C. nodulosa & CBS 160.58 & - & - & JN601033 & JN600975 & JN601019 \\
\hline C. oryzae & CBS 16953 & Oryza sativa & Vietnam & KP400650 & KP645344 & KM196590 \\
\hline C. ovariicola & BRIP 15882 & - & - & JN601031 & JN600971 & JN601020 \\
\hline C. pallescens & CBS 156.35 & Air & Java & KJ922380 & KM083606 & KM196570 \\
\hline C. papendorfii & BRIP 57608 & Acacia karroo & - & KJ415552 & KJ415395 & KJ415441 \\
\hline C. papendorfii & CBS308.67 & Acacia karroo & $\begin{array}{l}\text { South } \\
\text { Africa }\end{array}$ & KJ909774 & KM083617 & KM196594 \\
\hline C. perotidis & CBS 350.90 & Perotis rara & Cape York & JN192385 & JN601021 & JN601021 \\
\hline C. petersonii & BRIP 14642 & $\begin{array}{l}\text { Dactyloctenium } \\
\text { aegyptium }\end{array}$ & Australia & MH414905 & MH433650 & MH433668 \\
\hline C. pisi & CBS 190.48 & Pisum sativum & Canada & KY905678 & KY905690 & KY905697 \\
\hline C. platzii & $\begin{array}{l}\text { BRIP } \\
27703 b\end{array}$ & $\begin{array}{l}\text { Cenchrus } \\
\text { clandestinum }\end{array}$ & Australia & MH414906 & MH433651 & MH433669 \\
\hline C. portulacae & BRIP 14837 & Soil & - & KJ415554 & KJ415392 & KJ415439 \\
\hline C. portulacae & CBS 239.48 & $\begin{array}{l}\text { Portulaca } \\
\text { oleracea }\end{array}$ & USA & KJ909775 & KM083616 & KM230404 \\
\hline C. prasadii & CBS 143.64 & $\begin{array}{l}\text { Jasminum } \\
\text { sambac }\end{array}$ & India & KJ922373 & KM061785 & KM230408 \\
\hline C. protuberata & 5876 & Fragaria sp. & - & KT012665 & KT012626 & KT012587 \\
\hline C. protuberata & CBS 376.65 & $\begin{array}{l}\text { Deschampsia } \\
\text { flexuosa }\end{array}$ & UK & KJ922376 & KM083605 & KM196576 \\
\hline $\begin{array}{l}\text { C. } \\
\text { pseudobrachyspora }\end{array}$ & CPC 28808 & Eleusine indica & Thailand & MF490819 & MF490841 & MF490862 \\
\hline C. pseudolunata & $\begin{array}{l}\text { UTHSC } \\
092092\end{array}$ & Nasal sinus & USA & HE861842 & HF565459 & - \\
\hline C. pseudorobusta & $\begin{array}{l}\text { UTHSC } \\
083458\end{array}$ & Nasal sinus & USA & HE861838 & HF565476 & - \\
\hline C. ravenelii & BRIP 13165 & $\begin{array}{l}\text { Sporobolus } \\
\text { fertilis }\end{array}$ & Australia & JN192386 & JN600978 & JN601024 \\
\hline C. reesii & BRIP 4358 & Air & Australia & MH414907 & MH433637 & MH433670 \\
\hline
\end{tabular}


Table 1 Continued.

\begin{tabular}{|c|c|c|c|c|c|c|}
\hline \multirow[t]{2}{*}{ Species } & \multirow[t]{2}{*}{ Strain no." } & \multirow[t]{2}{*}{ Source } & \multirow[t]{2}{*}{ Origin } & \multicolumn{3}{|c|}{ GenBank Accession numbers } \\
\hline & & & & ITS & GPDH & EF1a \\
\hline C. richardiae & BRIP 4371 & $\begin{array}{l}\text { Richardia } \\
\text { brasiliensis }\end{array}$ & Australia & KJ415555 & KJ415391 & KJ415438 \\
\hline C. robusta & CBS624 68 & $\begin{array}{l}\text { Dichanthium } \\
\text { annulatum }\end{array}$ & USA & KJ909783 & KM083613 & KM196577 \\
\hline C. rouhanii & $\begin{array}{l}\text { CBS } \\
\text { 144674; } \\
\text { SCUA-2bi }\end{array}$ & $\begin{array}{l}\text { Syngonium } \\
\text { vellozianum }\end{array}$ & Iran & KX139030, & MG428694 & MG428687 \\
\hline C. rouhanii & $\begin{array}{l}\text { CBS } \\
\text { 144675; } \\
\text { IRAN } \\
\text { 3138C; } \\
\text { SCUA-4bi }\end{array}$ & Eucalyptus sp. & Iran & KX139032 & MG428696 & MG428689 \\
\hline C. ryleyi & BRIP 12554 & $\begin{array}{l}\text { Sporobolus } \\
\text { creber }\end{array}$ & - & KJ415556 & KJ415390 & KJ415437 \\
\hline C. ryleyi & CBS349.90 & $\begin{array}{l}\text { Sporobolus } \\
\text { creber }\end{array}$ & Australia & KJ909766 & KM083612 & KM196567 \\
\hline C. senegalensis & CBS 149.71 & - & - & HG779001 & HG779128 & - \\
\hline C. soli & CBS 222.96 & Soil & $\begin{array}{l}\text { Papua New } \\
\text { Guinea }\end{array}$ & KY905679 & KY905691 & KY905698 \\
\hline C. sorghina & BRIP 15900 & $\begin{array}{l}\text { Sorghum } \\
\text { bicolor }\end{array}$ & Australia & KJ415558 & KJ415388 & KJ415435 \\
\hline C. spicifera & CBS 274.52 & Soil & Spain & JN192387 & JN600979 & JN601023 \\
\hline C. sporobolicola & $\begin{array}{l}\text { BRIP } \\
23040 \mathrm{~b}\end{array}$ & $\begin{array}{l}\text { Sporobolus } \\
\text { australasicus }\end{array}$ & Australia & MH414908 & MH433652 & MH433671 \\
\hline C. subpapendorfii & CBS656.74 & Desert soil & Egypt & KJ909777 & KM061791 & KM196585 \\
\hline C. trifolii & AR5169 & $\begin{array}{l}\text { Sorghum } \\
\text { bicolor }\end{array}$ & $\begin{array}{l}\text { South } \\
\text { Africa }\end{array}$ & KP400656 & KP645345 & KP735694 \\
\hline C. tripogonis & BRIP 12375 & Unknown & Australia & JN192388 & JN600980 & JN601025 \\
\hline C. tropicalis & BRIP 14834 & Coffea arabica & India & KJ415559 & KJ415387 & KJ415434 \\
\hline C. tsudae & $\begin{array}{l}\text { BRIP } \\
10970 \mathrm{a}\end{array}$ & Chloris gayana & Australia & KC424605 & KC747755 & КС503940 \\
\hline C. tsudae & $\begin{array}{l}\text { MAFF } \\
236750\end{array}$ & Chloris gayana & Japan & KP400651 & KM061790 & KM230409 \\
\hline C. tuberculata & CBS 14663 & Zea mays & India & JX256433 & JX276445 & JX266599 \\
\hline C. uncinata & CBS 221.52 & Oryza sativa & Vietnam & HG779024 & HG779134 & - \\
\hline C. variabilis & СРC 28813 & $\begin{array}{l}\text { Digitaria } \\
\text { ciliaris }\end{array}$ & Thailand & MF490820 & MF490842 & MF490863 \\
\hline C. variabilis & CPC 28815 & $\begin{array}{l}\text { Chloris } \\
\text { barbata }\end{array}$ & Thailand & NR154866 & MF490844 & MF490865 \\
\hline C. verruculosa & CBS150 63 & $\begin{array}{l}\text { Punica } \\
\text { granatum }\end{array}$ & India & KP400652 & KP645346 & KP735695 \\
\hline C. verruculosa & $\begin{array}{l}\text { MFLUCC } \\
100690\end{array}$ & Oryza sativa & Thailand & JX256437 & JX276448 & JX266602 \\
\hline C. warraberensis & BRIP 14817 & $\begin{array}{l}\text { Dactyloctenium } \\
\text { aegyptium }\end{array}$ & Australia & MH414909 & MH433653 & MH433672 \\
\hline Curvularia sp. & AR 5117 & Lolium perenne & USA & KP400655 & КР645349 & KP735698 \\
\hline Curvularia sp. & BRIP 17439 & $\begin{array}{l}\text { Trianthema } \\
\text { Portulaca } \\
\text { strum }\end{array}$ & Australia & AF081449 & AF081406 & MH445455 \\
\hline Curvularia sp. & $\begin{array}{l}\text { MFLUCC } \\
100709\end{array}$ & Oryza sativa & Thailand & JX256442 & JX276453 & - \\
\hline Curvularia sp. & $\begin{array}{l}\text { MFLUCC } \\
100739\end{array}$ & Oryza sativa & Thailand & JX256443 & JX276454 & JX266603 \\
\hline Curvularia sp. & $\begin{array}{l}\text { MFLUCC } \\
120177\end{array}$ & Unknown grass & Thailand & KP400654 & КР645348 & KP735697 \\
\hline
\end{tabular}


Table 1 Continued.

\begin{tabular}{lllllll}
\hline Species & Strain no." & Source & Origin & & \multicolumn{2}{c}{ GenBank Accession numbers } \\
\cline { 5 - 7 } & & & & ITS & GPDH & EF1a \\
\hline Curvularia sp. & UTHSC & Human & USA & HE861826 & HF565477 & - \\
& 08809 & & & & & \\
\hline${ }^{*}$ BRIP: & Q & & & &
\end{tabular}

"BRIP: Queensland Plant Pathology Herbarium, Brisbane, Australia; CBS: Centraalbureau voor Schimmelcultures, Utrecht, The Netherlands; CPC: Culture collection of Pedro Crous, housed at Westerdijk Fungal Biodiversity Institute; DAOM: Plant Research Institute, Department of Agriculture (Mycology), Ottawa, Canada; ICMP: International Collection of Micro-organisms from Plants, Landcare Research, Private Bag 92170, Auckland, New Zealand; IMI: International Mycological Institute, Kew, UK; IRAN: Iranian Fungal Culture Collection, Iranian Research Institute of Plant Protection, Iran; MAFF: Ministry of Agriculture, Forestry and Fisheries, Tsukuba, Ibaraki, Japan, MFLUCC: Mae Fah Luang University Culture Collection, Chiang Rai, Thailand; UTHSC: Fungus Testing Laboratory, Department of Pathology at the University of Texas Health Science Center, San Antonio, Texas, USA; SCUA: the Collection of Fungal Cultures, Department of Plant Protection, Shahid Chamran University of Ahvaz, Iran.

Cultural characters - Colonies on PDA attaining 60-65 mm diam. after $8 \mathrm{~d}$ incubation at $28 \pm 0.5{ }^{\circ} \mathrm{C}, 48-52 \mathrm{~mm}$ diam. at $35 \pm 0.5{ }^{\circ} \mathrm{C}$ and $9-9.4 \mathrm{~mm}$ diam. at $40 \pm 0.5{ }^{\circ} \mathrm{C}$, circular with entire margin, greenish grey (green jade) with colorless edge, paler with age, aerial mycelium sparse to abundant, floccose with age; reverse greenish black, paler towards the edge of the colony, with age becoming darker. Colonies on CMA, $80-84 \mathrm{~mm}$ diam. after $8 \mathrm{~d}$ incubation at $28 \pm 0.5{ }^{\circ} \mathrm{C}$, looser than on PDA, circular with entire margin, blackish green with paler edge, paler with age, aerial mycelium sparse to abundant, with age slightly floccose; reverse greenish black, paler and greener towards the edge of the colony, with age becoming darker.

Habitat - common zinnia.

Distribution - Iran (Ahvaz).

Notes - In the phylogenetic analysis of combined ITS-GPDH-EF1 $\alpha$ dataset (Fig. 1), Curvularia ahvazensis is phylogenetically related to C. dactyloctenii (Alcorn) Y.P. Tan \& R.G. Shivas (Alcorn 1982, Tan et al. 2014, 2018), C. hawaiiensis (Bugnic.) Manamgoda, L. Cai \& K.D. Hyde (Manamgoda et al. 2012), and C. nodosa Y. Marín, Cheew. \& Crous (Marin-Felix et al. 2017b). The length of $C$. ahvazensis conidia (13.5-27 $\mu \mathrm{m})$ is similar to those of $C$. nodosa (14.5-25 $\mu \mathrm{m}$ ), while the conidia of $C$. dactyloctenii (32-55 $\mu \mathrm{m}$ long) and $C$. hawaiiensis (12-37 $\mu \mathrm{m}$ long) are longer. The conidia of $C$. ahvazensis (5-7.8 $\mu \mathrm{m}$ wide) and $C$. dactyloctenii (6-8 $\mu \mathrm{m}$ wide) are narrower than those of $C$. hawaiiensis $(8-10 \mu \mathrm{m}$ wide) and $C$. nodosa (7-9.5 $\mu \mathrm{m}$ wide). Conidiophores of $C$. ahvazensis are 2-4 $\mu \mathrm{m}$ wide, while in $C$. hawaiiensis and $C$. nodosa they are 2-6 $\mu \mathrm{m}$ and 3-5.5 $\mu \mathrm{m}$ wide, respectively.

Curvularia rouhanii M. Mehrabi-Koushki \& G. Babaahmadi, sp. nov.

Fig. 3

MycoBank: MB823474

Typification - IRAN, KHUZESTAN PROVINCE: Ahvaz. On blighted leaves of syngonium (Syngonium vellozianum), Oct 2015, G. Babaahmadi and M. Mehrabi-Koushki (holotype: IRAN 17006F, ex-type cultures: CBS $144674=$ SCUA-2bi-2).

Etymology - In reference to the significant contribution of Prof. Hamid Rouhani to develop biological control of plant diseases in Iran.

Morphology on PDA - Hyphae hyaline to pale brown, poorly branched, septate, occasionally with pear-shaped swollen cells, 2-6 $\mu \mathrm{m}$ diam. Conidiophores arising singly, septate, generally equal wide in median or upper parts, base thinner with a narrowed basal cell, straight or flexuous, apically geniculate, un-branched, cell walls thicker than those of vegetative hyphae, brown, paler towards apex, $(17.5-) 25-155(-230) \times 2.5-5(5.5) \mu \mathrm{m}, 95 \%$ confidence limits $=63.9-83.2 \times 3.8-4.1$ $\mu \mathrm{m},(\overline{\mathrm{x}} \pm \mathrm{SD}=73.6 \pm 47.6 \times 4 \pm 0.8 \mu \mathrm{m}, \mathrm{n}=50)$. Conidiogenous cells integrated or discrete, smooth or with verruculose nodes, terminal or intercalary, proliferating sympodially, with circular and thickened scars, brown to dark brown, subcylindrical to swollen. Conidia smooth-walled, straight or very slightly curved, ellipsoid to cylindrical, tapering towards rounded ends, pale brown 


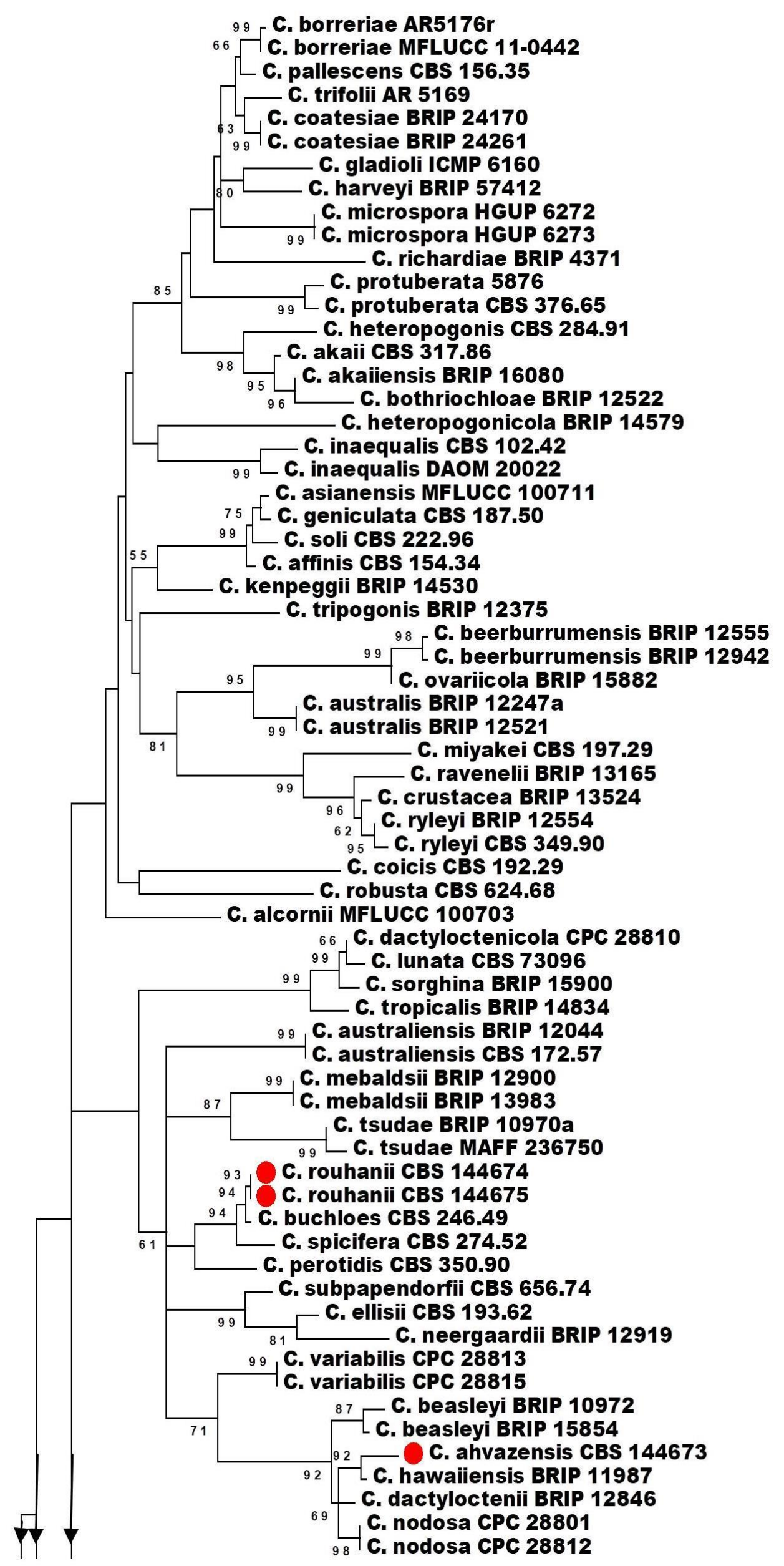




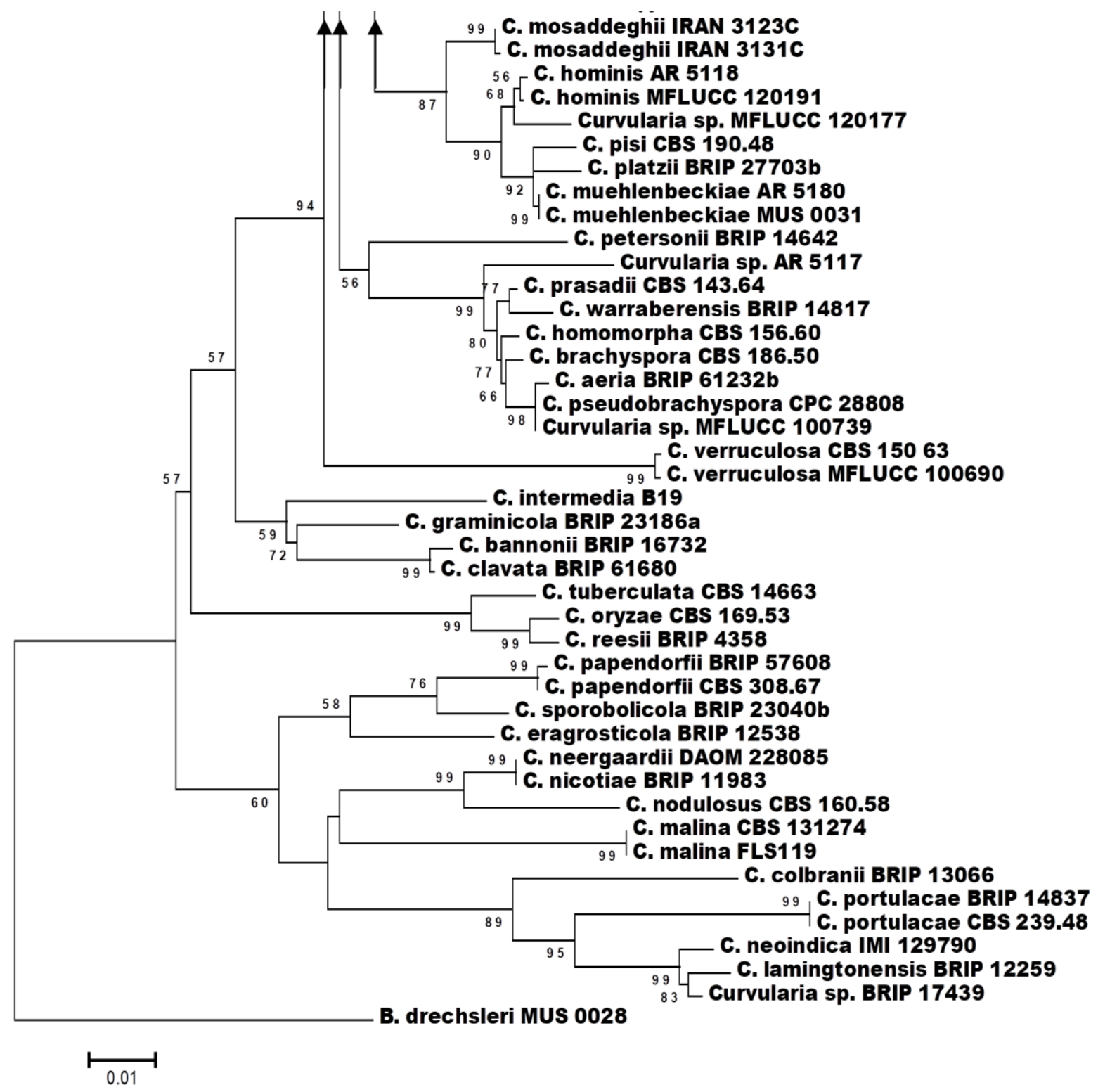

Figure 1 - Phylogenetic tree generated from a ML analysis based on a concatenated alignment of ITS, GPDH and EF1 $\alpha$ sequences of Curvularia strains representing most previously known species and new taxa. The tree was rooted with Bipolaris drechsleri MUS0028. Bootstrap values greater than $50 \%$ (expressed as percentages of 1000 replications) are shown at the nodes.

to brown, (2-)4-distoseptate, end cells not paler, 15-30.5 $\times(5.3-) 6.5-10 \mu \mathrm{m}, 95 \%$ confidence limits $=21.6-23.2 \times 8.3-8.7 \mu \mathrm{m},(\overline{\mathrm{x}} \pm \mathrm{SD}=22.4 \pm 4 \times 8.5 \pm 1 \mu \mathrm{m}, \mathrm{n}=50)$. Hila mostly inconspicuous sometime very slightly protruding, darkened, thickened, 2-3 $\mu \mathrm{m}$ diam. Chlamydospores unicellular or multicellular (pseudosclerotioid), smooth, dark brown to black. Swollen cells hyaline to pale brown, intercalary, mainly in clusters, variable in size and shape. Sexual morph not observed.

Cultural characters - Colonies on PDA attaining 50-55 mm diam. after $8 \mathrm{~d}$ incubation at $28 \pm 0.5{ }^{\circ} \mathrm{C}, 84-88 \mathrm{~mm}$ diam. at $35 \pm 0.5^{\circ} \mathrm{C}$ and $16-18 \mathrm{~mm}$ diam. at $40 \pm 0.5{ }^{\circ} \mathrm{C}$, circular with slightly undulate margin, greenish brown with colorless edge, paler with age, aerial mycelium abundant, highly floccose with age; reverse greenish black, blacker with age. Colonies on CMA, 82-86 mm diam. after $8 \mathrm{~d}$ of inoculation at $28 \pm 0.5{ }^{\circ} \mathrm{C}$, looser than on PDA, circular with entire margin, pale brown with lighter edge, paler with age, aerial mycelium sparse, slightly floccose with age; reverse pale brown with lighter edge. 
Additional cultures examined - IRAN, KHUZESTAN PROVINCE: Behbahan. From Eucalyptus sp., Oct 2015, G. Babaahmadi and M. Mehrabi-Koushki (CBS 144675 = IRAN 3138C).

Habitat - Syngonium and Eucalyptus.

Distribution - Iran (Ahvaz and Behbahan).

Notes - Phylogenetic analyses indicate that this species is closely related to C. buchloes (Lefebvre \& A.G. Johnson) Manamgoda, Rossman \& K.D Hyde and C. spicifera (Bainier) Boedijn (Fig. 1), but is morphologically distinct. Curvularia rouhanii can be easily distinguished from $C$. buchloes (Manamgoda et al. 2015) by the production of shorter conidia with fewer septa [(2-)4distoseptate and 15-30.5 $\mu \mathrm{m}$ long in $C$. rouhanii vs. 3-8-distoseptate and (34-)40-69(-71) $\mu \mathrm{m}$ long in $C$. buchloes]. Furthermore, the conidia of $C$. buchloes are more curved than those of $C$. rouhanii (Manamgoda et al. 2015). The conidia of $C$. rouhanii differ from $C$. spicifera (Bainier 1908) in having more septa, and shorter and narrower conidia ( $C$. spicifera are predominantly 3-distoseptate and 30-36 × 11-14 $\mu \mathrm{m}$ ).
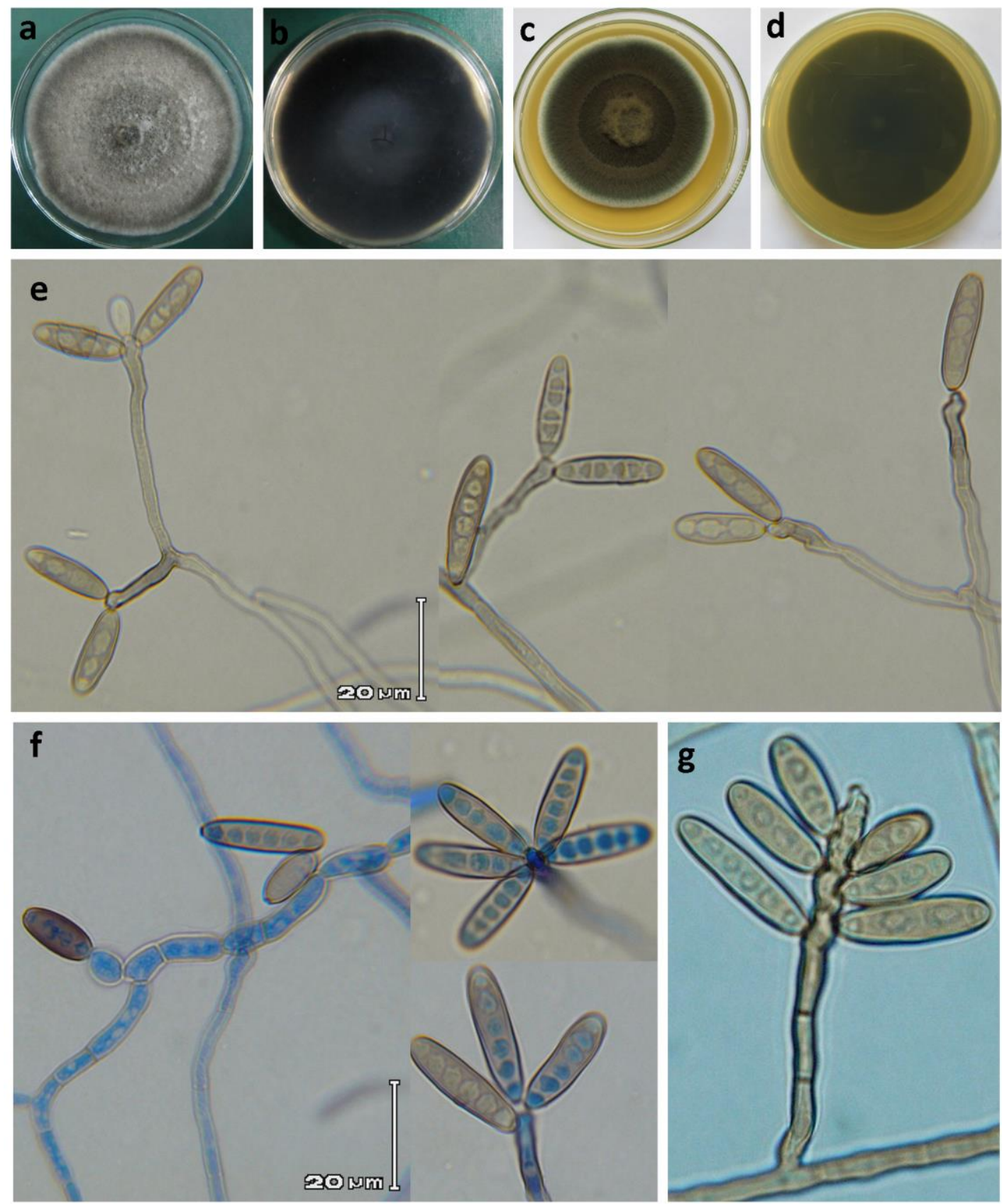

Figure 2 - Curvularia ahvazensis (CBS 144673). a-b Colony on PDA (top and reverse). c-d Colony on CMA (top and reverse). e-g Conidiophores, conidiogenous cells and conidia. 

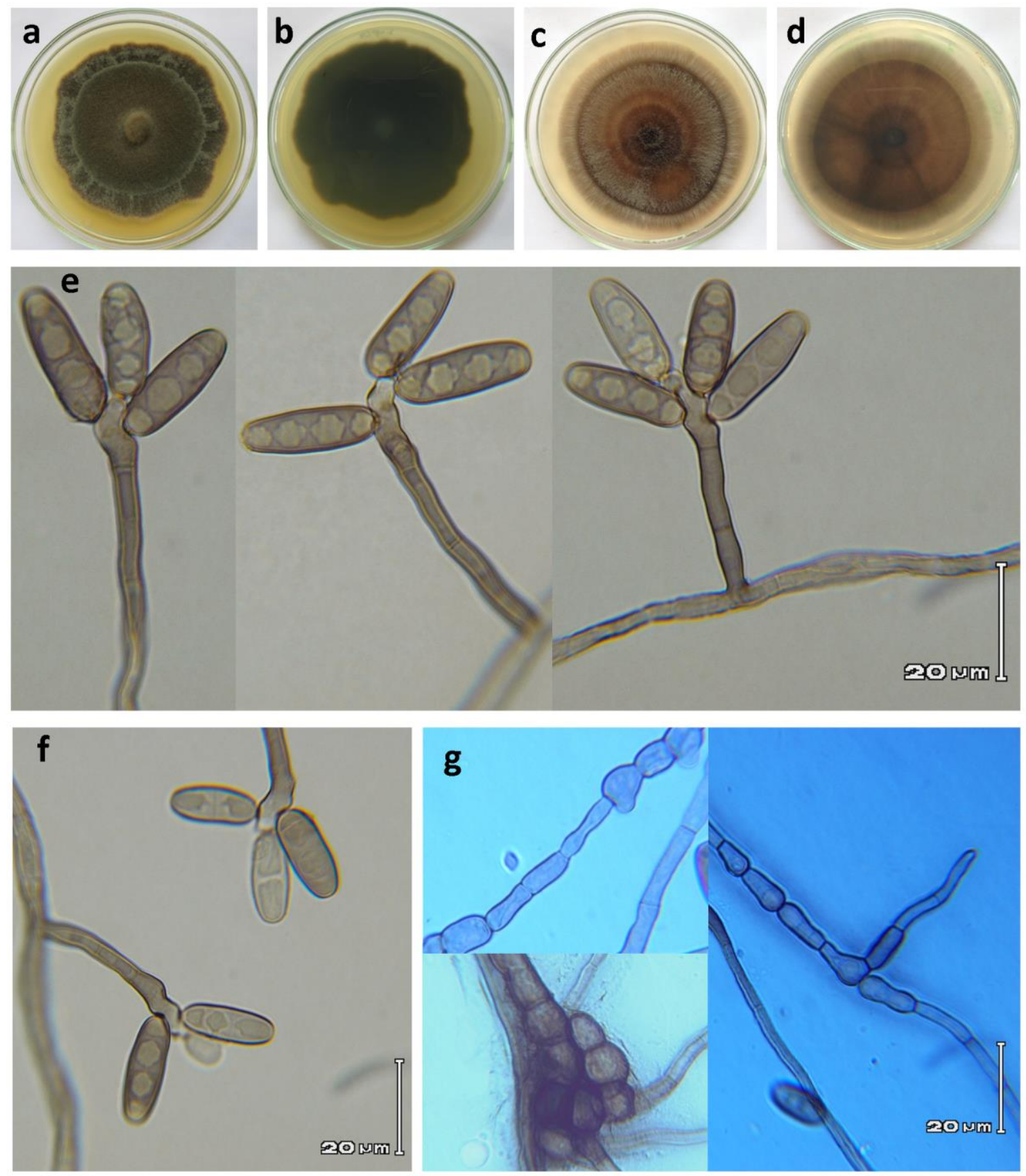

Figure 3 - Curvularia rouhanii (CBS 144674). a-b Colony on PDA (top and reverse). c-d Colony on CMA (top and reverse). e-f Conidiophores, conidiogenous cells and conidia. $\mathrm{g}$ Swollen cells and multicellular chlamydospores.

\section{Discussion}

The combination of phylogenetic analysis with morphological characterization showed that both Curvularia ahvazensis and C. rouhanii, are novel species. In both two- and three-locus-based phylogenetic trees, the combined datasets separated both new taxa from previously known species of Curvularia. In addition to their phylogenetic delimitation, morphological differences, including conidial size, shape and septation, can distinguish them from their closest relatives among the Curvularia species. Phylogenetic trees obtained from maximum likelihood analysis in this study yielded the same overall topology as that presented in previous surveys (Manamgoda et al. 2012a, b, 2014, 2015, Tan et al. 2014, 2018, Tomaso-Peterson et al. 2016, Marin-Felix et al. 2017a, b, Heidari et al. 2018, Liang et al. 2018). 
Curvularia species are commonly isolated from soil and living or dead parts of plants (Manamgoda et al. 2012a, b, 2014, 2015, Tan et al. 2014, 2018, Ariyawansa et al. 2015, TomasoPeterson et al. 2016, Marin-Felix et al. 2017a, b, Wijayawardene et al. 2017, 2018, Heidari et al. 2018, Liang et al. 2018). Some species have been also found in human tissues (Manamgoda et al. 2012a, da Cunha et al. 2013, Madrid et al. 2014). Curvularia ahvazensis was isolated from rotten roots of Zinnia elegans while $C$. rouhanii was isolated from blighted leaves of Syngonium vellozianum and Eucalyptus. Zinnia elegans was previously reported to be host of $C$. hawaiiensis (Manamgoda et al. 2015). Eucalyptus was known to be host of $C$. eragrostidis, $C$. hawaiiensis, $C$. pallescens and $C$. tuberculata (Manamgoda et al. 2011, 2015). This is the first report of a Curvularia species obtained from Syngonium vellozianum. Several species of Curvularia have been previously reported on herbaceous and woody plants from different areas of Iran, including $C$. affinis, $C$. australiensis, C. clavata, $C$. hawaiiensis, C. inaequalis, $C$. lunata, C. mosaddeghii, $C$. pallescens, $C$. papendorfii, $C$. prasadii, $C$. senegalensis, $C$. trifolii, $C$. tuberculata and $C$. verruculosa (Ershad 2009, Ahmadpour et al. 2013, Heidari et al. 2018, https://www.ncbi.nlm.nih.gov).

\section{Acknowledgment}

This work was financially supported by grants from Research Council of Shahid Chamran University of Ahvaz.

\section{References}

Ahmadpour A, Heidarian Z, Karami S, Pordel A et al. 2013 - New species of Bipolaris and Curvularia on poaceous plants in Iran. Rostaniha 14, 216-228.

Ahmadpour SA, Mehrabi-Koushki M, Farokhinejad R. 2017 - Neodidymelliopsis farokhinejadii, a new fungal species from dead branches of trees in Iran. Sydowia 69,171-182.

Alcorn JL. 1982 - New Cochliobolus and Bipolaris species. Mycotaxon 15, 1-19.

Alexopoulos CJ, Beneke ES. 1962 - Laboratory manual for introductory mycology. Burgess Pub. Co, Minneapolis.

Ariyawansa HA, Thambugala K, Manamgoda DS, Jayawardena R et al. 2015 - Towards a natural classification and backbone tree for Pleosporaceae. Fungal Diversity 71, 85-139.

Bainier MG. 1908 - Mycotheque de l'Ecole de Pharmacie. XXIV-XXVII. Bulletin de la Société Mycologique de France 24, 73-94.

Beneke ES, Rogers AL. 1996 - Medical mycology and human mycoses. Star Publishing Company, Belmont, 239 pp.

Berbee M, Pirseyedi M, Hubbard S. 1999 - Cochliobolus phylogenetics and the origin of known, highly virulent pathogens, inferred from ITS and glyceraldehyde-3-phosphate dehydrogenase gene sequences. Mycologia 91, 964-977.

Boedijn KB. 1933 - Ueber einige phragmosporen Dematiazeen. Bulletin du Jardin botanique de Buitenzorg 13, 120-134.

da Cunha KC, Sutton DA, Fothergill AW, Gene J et al. 2013 - In vitro antifungal susceptibility and molecular identity of 99 clinical isolates of the opportunistic fungal genus Curvularia. Diagnostic Microbiology and Infectious Disease 76, 168-174.

Ershad D. 2009 - Fungi of Iran, 3rd edn. Iranian Research Institute of Plant Protection, Tehran

Hall TA. 1999 - BioEdit: a user-friendly biological sequence alignment editor and analysis program for windows 95/98/NT. Nucleic Acids Symposium Series 41, 95-98.

Heidari K, Mehrabi-Koushki M, Farokhinejad R. 2018 - Curvularia mosaddeghii sp. nov., a novel species from the family Pleosporaceae. Mycosphere 9, 635-646.

Hibbett DS, Binder M, Bischoff JF, Blackwell M et al. 2007 - A higher-level phylogenetic classification of the Fungi. Mycological Research 111, 509-547.

Liang Y, Ran SF, Bhat J, Hyde KD et al. 2018 - Curvularia microspora sp. nov. associated with leaf diseases of Hippeastrum striatum in China. MycoKeys 29, 49-61. 
Madrid H, da Cunha KC, Gene J, Dijksterhuis J et al. 2014 - Novel Curvularia species from clinical specimens. Persoonia 33, 48-60.

Manamgoda DS, Cai L, Bahkali AH, Chukeatirote E et al. 2011 - Cochliobolus: an overview and current status of species. Fungal Diversity 51, 3-42.

Manamgoda DS, Cai L, McKenzie EHC, Crous PW et al. 2012a - A phylogenetic and taxonomic re-evaluation of the Bipolaris-Cochliobolus-Curvularia complex. Fungal Diversity 56, 13144.

Manamgoda DS, Cai L, McKenzie EHC, Chukeatirote E et al. 2012b - Two new Curvularia species from northern Thailand. Sydowia 64, 255-266.

Manamgoda DS, Rossman AY, Castlebury LA, Chukeatirote E et al. 2015 - Taxonomic and phylogenetic re-appraisal of the genus Curvularia (Pleosporaceae): human and plant pathogens. Phytotaxa 212, 175-198.

Manamgoda DS, Rossman AY, Castlebury LA, Crous PW et al. 2014 - The genus Bipolaris. Studies in Mycology 79, 221-288.

Marin-Felix Y, Groenewald JZ, Cai L, Chen Q et al. 2017a - Genera of phytopathogenic fungi: GOPHY 1. Studies in Mycology 86, 99-216.

Marin-Felix Y, Senwanna C, Cheewangkoon R, Crous PW. 2017b - New species and records of Bipolaris and Curvularia from Thailand. Mycosphere 8, 1556-1574.

O'Donnell K. 1993 - Fusarium and ITS near relatives. In: Reynolds DR, Taylor JW (eds) The fungal holomorph: mitotic, meiotic and pleomorphic speciation in fungal systematics. CABI Publishing, Wallingford, pp 225-233.

Raeder U, Broda P. 1985 - Rapid preparation of DNA from filamentous fungi. Letters in Applied Microbiology 1, 17-20.

Schoch C, Crous PW, Groenewald J, Boehm E et al. 2009 - A class-wide phylogenetic assessment of Dothideomycetes. Studies in Mycology 64, 1-15.

Tamura K, Stecher G, Peterson D, Filipski A, Kumar S. 2013 - MEGA6: molecular evolutionary genetics analysis version 6.0. Molecular Biology and Evolution 30, 2725-2729.

Tan YP, Madrid H, Crous PW, Shivas RG. 2014 - Johnalcornia gen. et. comb. nov., and nine new combinations in Curvularia based on molecular phylogenetic analysis. Australasian Plant Pathology 43, 589-603.

Tan YP, Crous PW, Shivas RG. 2018 - Cryptic species of Curvularia in the culture collection of the Queensland Plant Pathology Herbarium. MycoKeys 35, 1-25.

Tomaso-Peterson M, Jo YK, Vines PL, Hoffmann FG. 2016 - Curvularia malina sp. nov. incites a new disease of warm-season turfgrasses in the southeastern United States. Mycologia 108, 915-924.

Webster J, Weber RWS. 2007 - Introduction to Fungi, 3rd Ed. Cambridge University Press, Cambridge, UK.

White TJ, Bruns T, Lee S, Taylor J. 1990 - Amplification and direct sequencing of fungal ribosomal RNA genes for phylogenetics. In: Innis MA,Gelfand DH, Sninsky JJ, White TJ (eds) PCR protocols: a guide to methods and applications. Academic Press, New York, pp 315-322.

Wijayawardene NN, Hyde KD, Rajeshkumar KC, Hawksworth DL et al. 2017 - Notes for genera: Ascomycota. Fungal Diversity 86, 1-594.

Wijayawardene NN, Hyde KD, Lumbsch T, Liu JK et al. 2018 - Outline of Ascomycota - 2017. Fungal Diversity 88, 167-263. 